\title{
Das Haupt des Appius
}

\author{
F. X. Ryan
}

\begin{abstract}
It is shown that the attestation of the principate of Ap. Claudius Pulcher (Cos. 143) is due to the alchemistic procedure of ancient writers. Other ostensible facts about Appius are also examined: a supposed testimonium for his censorship, his supposedly fixed date of death, and his supposed augurate.
\end{abstract}

\section{J. LINDERSKI} septuagenario

Bei der spärlichen Überlieferung schaut kein Althistoriker gern einem geschenkten

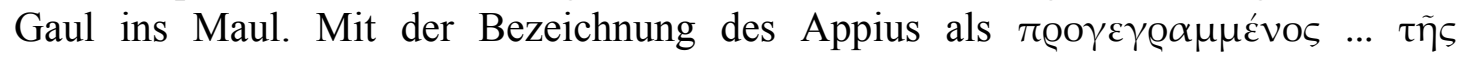

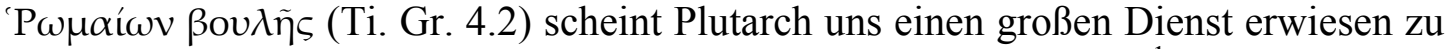
haben, denn dessen Prinzipat wird an keiner zweiten Stelle erwähnt. ${ }^{1}$ An und für sich erweckt die Einzahl der Belegstellen keinen Verdacht. Sieht man einmal von Appius ab, geht etwa ein Drittel der bezeugten Prinzipate - vier von elf - auf eine einzige Quelle zurück. Deren gibt es keins, das man ohne weiteres akzeptieren kann, doch lassen sich die vier allesamt durch Argumente retten, wobei allerdings zwei (das des Lupus und das des Tullius) dennoch nicht über allen Zweifel erhaben sind und zwei (das des Gurges und das des Corculum) leider auf inhaltlich fehlerhaften Passagen basieren. Es spricht also nicht entscheidend gegen das Prinzipat des Appius, daß der Beleg dafür chronologische Probleme aufweist, auch nicht, daß er sich in einer Erzählung befindet, die gelinde gesagt schon bei der ersten, flüchtigen Lektüre anekdotenhaft und möglicherweise unhistorisch wirkt, denn gerade erfundene Geschichten müssen der Glaubwürdigkeit halber ein gerüttelt Maß an Richtigem enthalten. Zudem steht außer Frage, daß Plutarch mit den oben zitierten Worten Appius als den Ersternannten des Senats bezeichnen wollte, denn er hat dasselbe Tätigkeitswort in Zusammenhang mit vier Männern (Scipio Africanus, Valerius, Lepidus und Scaurus) gebraucht, deren Vorrangstellung keinem Zweifel unterliegt. ${ }^{2}$

\footnotetext{
${ }^{1}$ T. Mommsen (1888) machte etwas widersprüchliche Aussagen zu dem Einfluß des Princeps Senatus: Er hatte „das wichtige Vorrecht des ersten Wortes bei allen Verhandlungen“ (3.969-70); später fand man sich nicht mehr ab ,mit der wenn auch nur nominellen Vorstandschaft eines einzelnen Mitgliedes“ (3.970). Es ist schwierig, den Princeps Senatus mit den beiden anderen, als primus rogatus Fungierenden zu vergleichen, denn im Falle des Princeps fehlen die zeitgenössischen Quellen, die über die ersten Anläufe berichtet hätten: „The late sources which narrate their participation probably overstate their rate of success in consequence" (Ryan 1998: 323-24; vgl. 343-44, 345 A. 370); die Principes scheinen sich ,somewhat more than one-third, but probably less than one-half, of the time“ durchgesetzt zu haben (Ryan 1998: 345). In gewisser Hinsicht ist die Untersuchung zu dem Einfluß des Prinzipats die Jagd nach einem Phantom: „Ultimately the principatus is an abstraction; it did not exist apart from individual principes... More evidence would allow us to say more about the influence of individual principes, but more evidence would not help us to say more...about the importance of the principatus“ (Ryan 1998: 356). Unlängst ist der Zeitraum, in dem Principes Senatus dienten bzw. später gedient zu haben schienen (dazu vgl. Ryan 1998: 280-83), präzisiert worden: Sieht man von der möglichen Ernennung des Cicero ab, liegen zwischen dem ersten und dem letzten Princeps um die 190 Jahre; die Stellung entstand offenbar i. J. 265 v. Chr. (vgl. Ryan 2003: 112-13) und erlosch ca. 75 v. Chr. (s. Ryan 2002: 380-82).

${ }^{2}$ Vgl. Ryan 1998: 172.
} 
Mit einem Wort: Es überrascht nicht, daß sich Appius bislang als der Ersternannte des Senats behaupten konnte.

Allgemein bekannt ist die Geschichte, nach welcher ein Brautvater seiner Gattin mitteilt, er habe die Tochter verlobt, woraufhin sie sich erzürnt: Keine Rücksicht sei auf sie genommen worden; ihr Mann habe das nicht tun dürfen, selbst wenn der Verlobte Ti. Gracchus heiße. Da ist der Familienvater außer sich vor Freude, denn ausgerechnet mit Tiberius hatte er gerade die Tochter verlobt. In den lateinischen Quellen (L. 38.57.2-8, ${ }^{3}$ Val. Max. 4.2.3, Gell. 12.8.2-4) ist der Schwiegervater P. Scipio Africanus und der Schwiegersohn der ältere Tiberius Gracchus, Livius (38.57.3) war aber wenigstens mit dem Einwand vertraut, das Verlöbnis habe man erst post mortem patris bekannt gegeben. Bei Plutarch dagegen ist der Schwiegervater Ap. Claudius Pulcher und der Schwiegersohn der jüngere Tiberius Gracchus. Nun hielt Plutarch die andere Fassung nicht geheim; er wisse, bei $\tau \iota v \varepsilon \varsigma$ werde dasselbe von Tiberius, dem Vater der Gracchi, und Scipio Africanus erzählt, bei oi $\pi \lambda$ cíouৎ aber werde das weitergegeben, was er aufzeichne (Ti. Gr. 4.4). Dann erbringt Plutarch einen zwingenden Beweis. Polybios berichte, erst nach Scipios Tode habe die

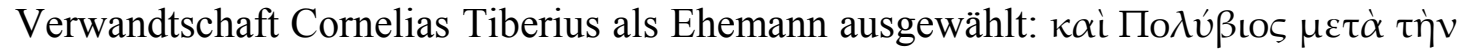

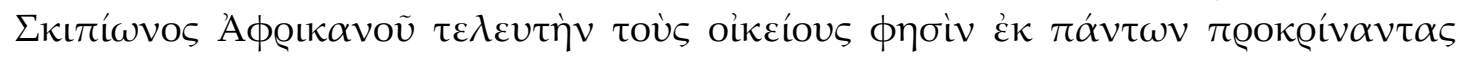

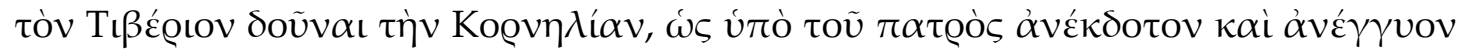

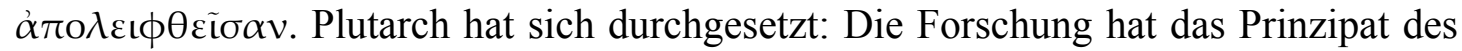
Appius nie in Zweifel gezogen, sondern die Plutarchstelle als den Nachweis der Ernennung unbekümmert angegeben ${ }^{4}$ oder sich allenfalls mit dem Datum der Ernennung befaßt. ${ }^{5}$

„Beglaubigt ist natürlich keine der beiden Versionen; doch wird die...Fassung, die Plutarch für die gangbarere erklärt und die nicht wie die andere gegen feststehende Thatsachen verstößt, die ältere sein." ${ }^{\text {6 }}$ Doch verstößt auch die Version Plutarchs „gegen feststehende Thatsachen,“ da die Hochzeit spätestens Mitte 136 erfolgt ist: Appius war entgegen der Aussage Plutarchs zu jenem Zeitpunkt noch kein censorius, und höchstwahrscheinlich weder Zensor noch Vormann des Senats zur Zeit der Verlobung. ${ }^{7}$ Die Behauptung, die der Schreibende an anderer Stelle aufgestellt hat „Plutarch...was right to dissociate the story from Gracchus senior, since Polybius has another version of that betrothal ${ }^{\text {* } 8}$ — setzt voraus, daß die eine oder die andere Version richtig ist. Auf ähnliche Weise zog Mommsen den Schluß, daß die Version, in welcher Appius als der Schwiegervater auftritt, wenn auch nicht die richtigere, so doch ,die ältere" Version sein müsse, da die Version, in welcher Africanus, entgegen „der wahrhaften und schönen Erzählung des Polybios,“ als der Schwiegervater auftritt, nicht richtig sein könne. ${ }^{9}$ Streng genommen aber hat Polybios im Vorhinein

\footnotetext{
${ }^{3}$ Bei Livius (38.57.7: cum illa...adiecisset, non, si Ti. Graccho daret, expertem consilii debuisse matrem esse) könnte man zwar den Text zu non, <ni>si verbessern (so Konrad 1989: 155-57), aber auch weiterhin $s i$ als etsi auffassen.

${ }^{4}$ Drumann 1835: 2.181 A. 71; Münzer 1899: 2848.

${ }^{5}$ Mommsen (1864: 1.93 A. 51, 94): „wahrscheinlich...618 [=136 v. Chr.]“; Willems (1878: 1.113 A. 4 , 6): „Appius peut n'avoir été nommé qu'en 131"; Ryan (1998: 185): „The principatus...is attested by Plutarch alone, but the fact...has never been doubted.... The date...has been a subject of dispute."

${ }^{6}$ Mommsen 1879: 2.493 A. 158.

${ }^{7}$ Vgl. Ryan 1998: 185.

${ }^{8}$ Ryan 1998: 185 A. 94

${ }^{9}$ Mommsen 1879: 2.492. Vgl. Konrad (1989: 156): „Plutarch's tale“ ist „a story generally agreed to be the model for the anecdote in Livy."
} 
die Historizität der Version bei Livius völlig widerlegt, ohne die Version Plutarchs untermauert zu haben. ${ }^{10}$ Es steht zunächst jedenfalls zu hoffen, daß eine der beiden Versionen richtig ist. Es versteht sich aber von selbst, daß die spätere Version nicht richtig sein kann, wenn schon die ursprüngliche Version eine glatte Erfindung ist, und es steht fest, daß die weniger geläufige Version nicht historisch ist. M. a. W.: Es ergäbe sich mit Sicherheit, daß auch die Version bei Plutarch falsch ist, wenn sich der Schluß ziehen ließe, daß die Version bei Livius älter ist.

Mommsen meinte: „Die Verschiebung lag freilich nahe, bringt aber eine arge Unschicklichkeit in die Erzählung; denn wie konnte Tiberius Gracchus, nachdem er eben sich öffentlich und feierlich verschworen hatte trotz der Intercession zu Gunsten des L. Scipio demselben feind bleiben zu wollen, unmittelbar darauf durch die Verlobung mit der Nichte desselben sich selber Lügen strafen?"11 Mommsen erklärte nicht, was genau er unter den Worten „Die Verschiebung lag freilich nahe“ verstand, und das ist kein Zufall, denn „freilich“ zeigt, daß er die Sache für selbstverständlich hielt. Der Schreibende kann an nur einen Grund denken, warum die Verschiebung „freilich“ nahegelegen hätte, nämlich die Tatsache, daß der eine Schwiegervater eines gewissen Ti. Gracchus ungleich wichtiger und berühmter war als der andere. Wenn aber irgend jemand im Zentrum der Geschichte steht, dann ist es der Bräutigam, und der gleichnamige Sohn erlangte durch sein jähes Ende eine Berühmtheit, welche die des Vaters übertraf, so daß eine Verschiebung in die entgegengesetzte Richtung, wenn man eher auf den Bräutigam als den Schwiegervater achtet, ohne weiteres möglich erscheint. Da Verschiebungen sowohl in die eine als auch in die andere Richtung vorstellbar sind, sind sie offensichtlich nicht der Weg, der zum Ziel führt.

Münzer meinte: „Die Erzählung von der Verlobung des jungen Augurs, die fälschlich auf seinen Vater übertragen wurde, ist so glaubwürdig, wie eine Anekdote nur sein kann..., denn ihre Voraussetzungen sind ganz und gar geschichtlich. “12 Die Verschiebungen stellten sich als eine Sackgasse heraus, vielleicht bieten die Voraussetzungen einen wirklichen Ausweg. Was gibt es nun an „ganz und gar“ geschichtlichen Voraussetzungen? In der weniger geläufigen Version haben Scipio und der ältere Tiberius bei einem Jupiterschmaus auf dem Capitol die Ehe vereinbart. In dieser Version ist nicht von dem Rang Scipios die Rede, während Tiberius (Tr. P1. 187?) gerade das Volkstribunat ausübte. In der weiter verbreiteten Variante spielt sich alles bei einem Antrittsschmaus ab. In dieser Version kann nicht mehr von dem Rang des Tiberius die Rede sein, da er soeben dem Knabenalter entwachsen sein soll (Plut. Ti.

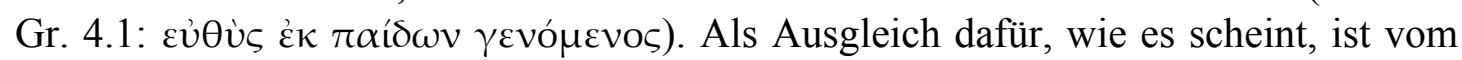

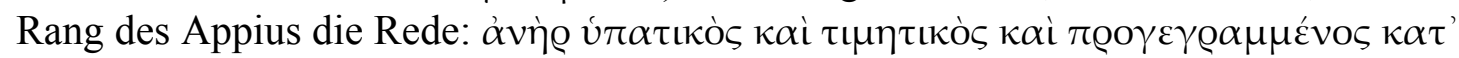

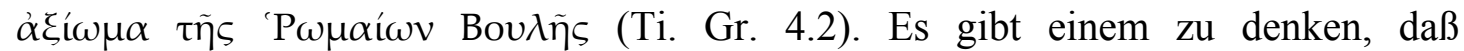
möglicherweise keine der drei Angaben den Rang des Appius zur Zeit der Verlobung richtig beschreibt, ${ }^{13}$ während alle drei nachweislich längst vor dem Jahr 187 im Falle Scipios zutreffend sind: Er war natürlich nach dem Jahr 205 consularis, ab dem Jahr 198 censorius und schon im Jahr 199 princeps senatus. Appius hätte seinerseits

\footnotetext{
${ }^{10}$ Vgl. Valgiglio 1963 z. St.: "Plutarco per dare fede all'episodio da lui narrato si limita a far constatare l'infondatezza dell'attribuzione di esso al padre di Tiberio; ma la non storicità della prima attribuzione non prova assolutamente quella della seconda, che potrebbe essere infondata come la prima"; Konrad (1989: 156 A. 5): "Whether the exchange between Appius and Antistia is historical is beyond recovery”; L. Burckhardt - J. von Ungern-Sternberg (1994: 102): Die Geschichte wird ,vielleicht mit besserem Recht" von dem jüngeren Ti. Gracchus erzählt.

${ }^{11}$ Mommsen 1879: 2.478 A. 129.

${ }^{12}$ Münzer 1920: 268 A. 1.

${ }^{13}$ Vgl. Ryan (1998: 185 A. 97): "If the wedding did take place in 143, when Appius was consul, the statement that he was a consular at the time of the betrothal would also be incorrect."
} 


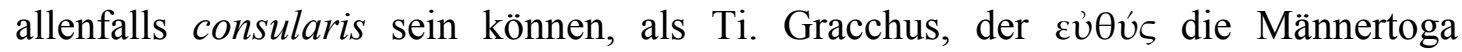
angelegt hatte, seine Aufnahme in das Auguralcollegium feierte. Damit scheinen wir die Frage nach der Richtung der Verschiebung beantwortet zu haben. Die ursprüngliche Version der Erzählung besetzte die Rolle des Schwiegervaters mit Scipio Africanus. Da diese Version aber, wie gesagt, in Hinblick auf die Verlobung nicht historisch ist, muß man auch den Priesterschmaus, während dessen Appius und der jüngere Tiberius die Ehe miteinander vereinbaren, für nicht historisch halten, denn die zweite Version stellt den Versuch dar, die erfundene Anekdote in eine wahre Anekdote zu verwandeln. Der Versuch war natürlich von vornherein zum Scheitern verurteilt. Der Erfinder der Anekdote glaubte sich aller Wahrscheinlichkeit nach mit der Rekonstruktion des Privatlebens des Africanus beschäftigt zu sein. Besser unterrichtet war er über die Ämterlaufbahn des Staatsmannes. Die Voraussetzungen, die ,ganz und gar geschichtlich" sind, sind eben diese drei Angaben zum Rang Scipios. Die spätere Version wirkt korrekt, weil sie eine nach besten Kräften korrigierte Version ist.

Der Zufall wollte es, daß die drei Angaben zum Rang des Schwiegervaters nur noch in der Version erhalten sind, in welcher diese Rolle mit Appius Claudius besetzt ist. Ihm darf man den Rang eines consularis nicht absprechen. Die Zensur des Appius ist gerade noch belegt. Broughton gibt dafür zwei Quellen an. Eine lange, verstümmelte Passage bei Festus (358/360/362L, s. v. religionis) behandelt mehrere Zensoren und erwähnt denjenigen der Zensoren des Jahres 136, der auch in den Fasti Capitolini genannt ist: ......... ${ }^{(16)}$........et $Q$. Fulvi No- $\mid<$ bilioris $>$... ${ }^{(6)} \ldots<$ Ap. Claudi Pulc $>$ ri fuit no- (S. 360, Z. 33-34, $r$ mit einem Unterpunkt versehen). Es scheint lediglich Wunschdenken zu sein, daß die Genitivendung des Kognomens des Appius hier erhalten ist. Leider ist die Passage so verstümmelt, daß die Namen der beiden Amtskollegen an keiner Stelle vollständig erhalten sind. Ein Bestandteil des Namens des Kollegen ist wahrscheinlich nur für die Zensoren des Jahres 154 v. Chr. erhalten: M. Vale<rius Messalla, C. Cassius Lon-> | ginus censores (Z. 19-20, e mit einem Unterpunkt versehen). In Zeile 34 liegen jedoch das fehlende Ende des Kognomens des Q. Fulvius und das vermeintliche Ende des Kognomens des Appius zu weit auseinander, wenngleich Müllers Einfallsreichtum (No<bilioris et eius collegae Ap. Claudii Pulc $>$ ri fuit no $<$ bilissima censura $>$ ) beeindruckend ist. Da nun et vor dem Namen des Q. Fulvius steht, kommt man unschwer auf den Gedanken, daß der Name des Appius nicht in Zeile 34, sondern in der großen Lücke in Zeile $33 \mathrm{zu}$ finden war. Dagegen spricht nicht $<$ A. Postu- $>\mid$ mius Q. Ful $<$ vius $>$ (Z. 2-3). Da in diesem Text Kognomina immer wieder begegnen (Pauli, Z. 13; P. Corneli Sc<ipionis $>$, Z. 16; $<$ Lon->ginus, Z. 19-20; Lentuli, Z. 26; Q. Fulvi No-<bilioris>, Z. 33-34; Metelli, Z. 36) und soweit feststellbar nie fehlen, so ist es alles andere als wahrscheinlich, daß der auf -mius ausgehende Name in Zeile 3 den Namen des Amtskollegen des Q. Fulvius Flaccus in der Zensur des Jahres 174, A. Postumius Albinus, darstellt. Es kommen einem daher Zweifel daran, ob hier der Name des Q. Fulvius im Nominativ steht. Wenn ja, dann erhebt sich die Frage, ob sich -mius nicht am Satzende befindet. Ebenso wenig spricht gegen unsren Vorschlag L. Corneli Lentuli C. $<$ Censorini $>$ (Z. 26), da der Amtskollege des L. Cornelius Lentulus Lupus in der Zensur des Jahres 147 kein Gaius, sondern ein Lucius war, nämlich L. Marcius Censorinus. Dessen Name gehört in die Lücke in Zeile 25. Man muß hier $<L$. Marci Censorini et $>\mid L$. Corneli Lentuli c $<$ ensura $>$ (Z. 25-26) lesen. ${ }^{14}$ Ein et gibt es auch am Anfang der 16.

\footnotetext{
${ }^{14}$ In Z. 26 behielt Lindsay nur $<$ Censorini $>$, während Müller $<$ Censorini sequitur censura $>$ ergänzt hatte.
} 
Zeile. Das -opilius am Anfang der 17. Zeile, ohne Kognomen, muß die zweite Erwähnung des Kollegen Corculums in der Zensur des Jahres 159 sein. Die erste Erwähnung gehört in die Lücke in Zeile 15. Hier muß man $<M$. Popili Laenatis $>\mid$ et P. Corneli Sc<ipionis $>$ (Z. 15-16) lesen. Dieses et begegnet uns wohl wieder bei der Zensur von 164: L. Aemili Pauli ae (Z. 13, a und $e$ mit einem Unterpunkt versehen). Die Lücke in der dieser vorangehenden Zeile ist groß genug, um dort den Namen des Kollegen des L. Paullus unterzubringen. Im Apparat aber liest man: ,e (i.e. ae) ut vid. $F^{\mathrm{L}}$ : e $F^{\mathrm{k}} F^{\mathrm{ab} ،}$. Es liegt nahe, hier L. Aemili Pauli e $<t$ Q. Marci Philippi $>$ (Z. 13) zu lesen. Die Zensoren von 154 werden bei Lindsay folgendermaßen vorgestellt: constat. M. Vale<rius Messalla, C. Cassius Lon-> | ginus censores (Z. 19-20, e mit einem Unterpunkt versehen). Der Platz fehlt nicht, ${ }^{15}$ die Namen der Amtskollegen hier wie sonst mit et miteinander zu verbinden. Höchstwahrscheinlich befand sich also der Name des Appius gänzlich in der vor et liegenden Lücke. Der Text ist wie folgt zu ergänzen: <Ap. Claudi Pulchri> et Q. Fulvi No- $\mid<$ bilioris $>$ (Z. 33-34). Damit fällt eine der zwei von Broughton angegebenen Belegstellen für die Zensur des Appius weg.

Es sei nun erlaubt, unsere Ergänzungen in benutzerfreundlicherer Weise bekannt zu machen. Die neuen Vorschläge werden unten in fetten Lettern gedruckt. Ein kritischer Text soll dieser selbstverständlich nicht sein.

\section{Festus 360L}

13 L. Aemili Pauli e $<$ t Q. Marci Philippi $>$

14 fuit, laboravit

15 amissione, capit $<$ is $>$

$<$ M. Popili Laenatis $>$

16 et $\mathrm{P}$. Corneli $\mathrm{Sc}<$ ipionis $>$

17 opilius, post cons

18 fuit vadatus

19 constat. M. Vale $<$ rius Messalla et C. Cassius Lon->

20 ginus censores $\mathrm{q}$

21 tia fuerat famosu

22 in Capitolio in ara

23 sico nata fuerat iu

24 tam ficum, infamesque

$<$ sine $>$

25 ullo pudicitiae respe $<$ ctu $><$ L. Marci Censorini et $>$

26 L. Corneli Lentuli $\mathrm{c}<$ ensura $>$

27 Lentulus iudicio pub $<$ lico $>$

28 fuerat. plurimi itaq $<$ ue $>$

29

30

31

$32<n a>$

33

$34<$ bilioris $>$

35

\author{
L. Mummi \\ ae in agen- \\ $<$ nul $>$ la soluta poe- \\ severitate \\ $<$ Ap. Claudi Pulchri> et Q. Fulvi No- \\ ri fuit no- \\ viribus notis
}

Die 25. Zeile enhält jetzt 39 Buchstaben und könnte vollständig sein.

\footnotetext{
${ }^{15}$ Wie von Lindsay ergänzt enthält die 19. Zeile 35 Buchstaben. Zum Vergleich geben wir die Anzahl der Buchstaben in den fünf letzten, vollständig erhaltenen Zeilen (S. 358, Z. 26-30) an: 40 litt., 35 litt., 37 litt., 47 litt., 40 litt. Es ist gut möglich, daß die so ergänzte 19. Zeile tatsächlich nur 34 Buchstaben hat: Da in diesem Eintrag Pauli (Z. 13) und opilius (Z. 17) begegnen, ist in Zeile 19 die Schreibweise Messala nicht auszuschließen.
} 
Die andere Stelle zählt zu den Excerpta Valesiana (V. 68, p. 621) aus Dio (23.81). Hier wird nicht einmal das Amt erwähnt, doch soll der zweite Mann der Kollege (ó $\sigma u v \alpha ́ \varrho \chi \omega v)$ des ersten gewesen sein. Die in Rede stehenden Amtsinhaber heißen ó

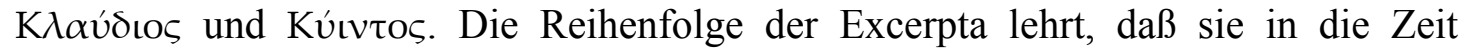
zwischen dem Konsulat des Q. Servilius Caepio (140 v. Chr.) und dem des L. Furius Philus (136 v. Chr.) gehören. Die Konsuln des genannten Zeitraumes sind natürlich bekannt und können nicht mit den Amtsträgern des Auszuges identifiziert werden. Ein Vergleich mit dem Auszug (V. 65, p. 618), der die Zensur des Jahres 142 referiert, beweist, daß Claudius und Quintus in der Tat als Zensoren amtiert haben. Auch hier wird das Amt mit keinem Wort erwähnt. In beiden Passagen werden die Amtskollegen in Hinblick auf Strenge einander gegenübergestellt: Damals

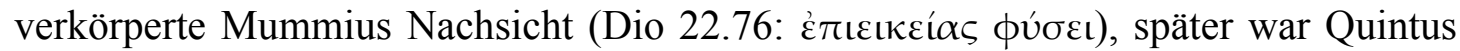

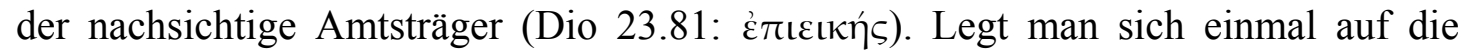
Zensur fest, kommt nur Appius infrage als der Kollege des Quintus. Appius darf also weiterhin als censorius gelten, obwohl es nur noch einen Beleg dafür gibt, und zwar einen, der lediglich seinen Gentilnamen angibt.

Es ist zumindest denkbar, daß Appius und Scipio Africanus nicht nur das Konsulat und die Zensur, sondern auch das Prinzipat gemeinsam haben. Die zweite Angabe, Appius sei censorius schon zur Zeit der Verlobung, ist nur anachronistisch. Dieser Zufall lehrt aber, daß diejenigen, die die Geschichte über Scipio zu berichtigen versuchten, nicht wußten, daß der jüngere Tiberius um die 10 Jahre vor der Zensur des Appius die Männertoga angelegt hatte. Wahrscheinlich wußten die Schriftsteller, daß Appius irgendwann Zensor war, sie wußten aber nicht genau wann. „A little learning is a dangerous thing." 16 Dieses "little" hat die Überlieferung dazu verführt, die Angaben über Scipio beizubehalten, anstatt sie zu hinterfragen. Ist also auch die dritte Angabe nur anachronistisch, aber nicht falsch, so wäre dies ein sehr großer Zufall. Es ist nämlich alles andere als wahrscheinlich, daß die Überlieferung, die nichts über das Datum der Zensur bzw. der Verlobung wußte, die Angabe zum Prinzipat auf seine Richtigkeit hin prüfte. Wir haben es nicht mit Geschichtswissenschaftlern zu tun. Profis wären ziemlich schnell dahintergekommen, daß die Volljährigkeit des Tiberius und die Zensur des Appius ein Jahrzehnt auseinander liegen. Wir haben es vielmehr mit Dilettanten zu tun, die sich gar keine Gedanken über die dritte Angabe machten oder sie allenfalls bemerkten und bei sich dachten: „Hoffen wir das Beste!“ Die Angabe zum Prinzipat wurde nicht deswegen beibehalten, weil man wußte, daß sie richtig war, sondern deswegen, weil man nicht wußte, daß sie falsch war. Zu Lebzeiten Plutarchs wußte man, sparsam mit Standbildern umzugehen. Bestieg ein neuer Kaiser den Thron, so ersetzte man nicht die ganze Statue, sondern man wechselte den Kopf aus. Die Plutarchstelle ist ein literarisches Beispiel für dieses Vorgehen. Den Kopf des Africanus schraubte man ab, in den Rumpf schraubte man alsbald den Kopf des Appius ein. Unklar bleibt, ob die Mehrzahl der Historiker, auf welche sich Plutarch berief, Scipios Personalien so ungeschickt weitergab, wie er es tat: Es steht fest, daß die Mehrheit Appius zum fraglichen Brautvater machte, es steht aber nicht fest, daß die Mehrheit ihn zum Ersternannten des Senats beförderte. ${ }^{17}$

\footnotetext{
${ }^{16}$ Alexander Pope, An Essay on Criticism (1711).

${ }^{17}$ Die Ansicht, nur ein Patrizier einer gens maior habe das Prinzipat übernehmen dürfen, ist ein Teil eines Zirkelschlusses; s. Ryan 1998: 225. Wenn es richtig gewesen wäre, die Liste der höheren
} 
Das Todesjahr des Appius löst sich damit von der Zensur der Jahre 131-130 ab. Die Behauptung „Appius died in $130^{\text {“18 }}$ stützt, wie es scheint, keine Quelle. Unter der Voraussetzung, daß Appius wie Lupus zum Ersternannten des Senats befördert worden wäre, wäre er wohl bis zum Jahr 130 gestorben, da nicht wahrscheinlich ist, daß Lupus danach ernannt wurde. Die Degradierung des Appius zum gewöhnlichen consularis bedeutet, daß Lupus möglicherweise schon im Jahre 136 in den ersten Senatssitz aufrückte. ${ }^{19}$ Die Degradierung wirkt sich sonst nicht auf unsere Vorstellung von dem Prinzipat aus, da keine sententia des Appius aus der Zeit des vermeintlichen Prinzipats uns bekannt ist. ${ }^{20}$ Der Tod des Appius hat sich später als der des Ti. Gracchus (App. BC 1.18), früher aber als der des jüngeren Africanus (Cic. Rep. 1.31) ereignet: Er ist also zwischen Ende 133 v. Chr. und Anfang 129 v. Chr. entschlafen. Bekanntlich hieß Appius die Agrarreform seines Schwiegersohnes gut. Diese seine Einstellung hilft uns heute weiter. Die Anzahl der Grenzsteine, die Appius als Kommissionsmitglied angeben, legt nahe, daß er jedenfalls nicht schon im Jahre 133 heimgegangen ist. $^{21}$

Cornelias Vater und Claudias waren beide gewesene Konsuln, gewesene Zensoren und Salier. Die Priesterwürde des Brautvaters wird aber weder in der einen noch in der anderen Version des Verlöbnisses erwähnt. In der Version der lateinischen Quellen speiste der ganze Senat auf dem Kapitol (L. 38.57.5: senatum eo die forte in Capitolio cenantem, V.M. 4.2.3: auctore senatu in Capitolio Iovis epulo, Gell. 12.8.2: cum...sollemni die epulum Iovi libaretur atque ob id sacrificium senatus in Capitolio epularetur), ${ }^{22}$ so daß Scipio nicht in seiner Eigenschaft als Salier, sondern als Senator

Geschlechter anhand der Liste der Principes Senatus zu rekonstruieren, müßten wir jetzt die Claudier aus der Liste der gentes maiores streichen, da wir den einzigen als solchen bezeugten Claudius aus der Liste der principes senatus soeben gestrichen haben.

${ }^{18}$ Ryan 1998: 185. Hier wurde die Aussage durch keinen Verweis auf eine Quelle oder die Sekundärliteratur bekräftigt. Offenbar geht das feste Datum 130 auf Broughton 1986 (Repr. d. Ausg. 1951-52) zurück, wo mehrmals ohne Fragezeichen dieses Jahr als das Todesjahr des Appius angegeben ist (Broughton 1986: 1.495 [bis], 503; 2.547 [bis]; 3.56, 188); ebenda wird sein Tod einmal ,in 131 or 130 “(1.501 A. 1) und einmal „before 129“ (1.503) datiert. Die Vorliebe für genaue Daten hindert einen daran, sie zu hinterfragen. Auch G. V. Sumner (1973: 17) übernahm 130 v. Chr. als ein festes Todesjahr.

${ }^{19}$ Siehe Ryan 2003: 113. Es wird damit, wenn auch nicht wahrscheinlich, so doch wahrscheinlicher, daß schon die Zensoren von 131 P. Cornelius Lentulus zum Princeps Senatus ernannten. Es ist zwar nicht mehr wahrscheinlich, daß P. Lentulus, selbst wenn er erst i. J. 125 ernannt wurde, fast fünf Jahre lang vor der Ernennung als primus rogatus fungierte, es bleibt aber möglich oder sogar wahrscheinlich, daß er lange genug so fungierte, um einen gewissen Anspruch auf die Ernennung zum Princeps Senatus zu haben. Die Erklärung für seinen Aufstieg kann ohnehin unverändert beibehalten werden: „The best explanation of his appointment is that the censors...gave the title princeps senatus to the man who was already first on the list by virtue of his seniority among ex-consuls" (Ryan 1998: 241).

${ }^{20}$ Was an anderer Stelle (Ryan 1998: 353 m. A. 423, vgl. 346 A. 380) zu sententiae über Verwandte gesagt wurde, gilt noch, obwohl es u. a. auch am Beispiel dieses vermeintlichen Princeps Senatus veranschaulicht wurde, denn für sententiae über Verwandte war der Rang des Antragstellers nicht relevant.

${ }^{21}$ Auf die Relevanz der Grenzsteine (s. Broughton 1986: 1.495) machte mich brieflich J. von UngernSternberg aufmerksam.

${ }^{22}$ Zur Zeit der Republik wurde das epulum Iovis wohl nur am 13. November als Bestandteil der ludi plebeii ausgerichtet; vgl. Bernstein 1998: 285 m. A. 318, 286. Bernstein (1998: 161 A. 232, 285 A. 321) bezieht auch L. 38.57.5 auf das Iovis epulum, Livius scheint den Anlaß aber nicht gekannt zu haben; in seiner Vorlage könnte es sich um das Festessen, das anläßlich eines Triumphes gegeben wurde (vgl. die Rede des M. Servilius, L. 45.39.13: illae epulae senatus, quae nec privato loco nec publico profano, sed in Capitolio eduntur, utrum hominum voluptatis causa an deorum honoris 
dabei gewesen sein soll. Vielleicht war der ältere Tiberius Gracchus damals noch kein Senator, er war aber wenigstens ein amtierender Volkstribun. Das Festessen machte Schwierigkeiten, als die Überlieferung die Erzählung zu berichtigen versuchte. Die gemeinsame Priesterwürde nutzte nichts, da Scipio in der unhistorischen Urversion nicht in seiner Eigenschaft als Salier anwesend war. Die mit der Arbeit des Polybios vertrauten Schriftsteller waren offenbar der Überzeugung, daß Tiberius der Sohn jung verheiratet war bzw. früh seine Priesterstelle antrat, und zwar zu jung bzw. zu früh, als daß er schon zum Senator aufgestiegen wäre. Da Plutarchs Gewährsmänner die Urversion nicht als eine erfundene Anekdote aufgefaßt hatten, machten sie sich auf die Suche nach einem Priesterschmaus, an welchem der jüngere Tiberius hätte teilnehmen können. Leider ist das Augurat für den jüngeren Tiberius nicht anderweitig belegt: Wir müssen deshalb mit der Möglichkeit rechnen, daß Plutarchs Gewährsmänner es in der Not vermutet haben. Die Nachricht ist aber an und für sich glaubwürdig, da der ältere Tiberius Augur gewesen war. Plutarch selbst sah in dem

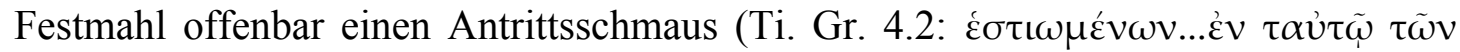

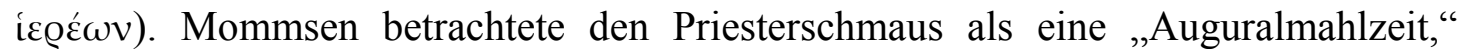
Münzer seinerseits als „,die Cena Aditialis des Tiberius.“23 Bardt hat das Augurat des jüngeren Ti. Gracchus übersehen, wie schon Münzer bemerkte, ${ }^{24}$ und damit verlor er die Gelegenheit, eine Stellungnahme zum Augurat des Appius abzugeben. Schon bei Drumann tritt Appius als Augur auf; Broughton datierte das Augurat ,?-130“ und erklärte: „A member of the college along with Ti. Gracchus, but when he entered is not known. ${ }^{, 25}$ Broughton scheint nicht erkannt zu haben, daß er darin mit Münzer uneins war: Dieser erwähnte das Augurat zwar in der Realencyclopädie mit keinem Wort, lehnte es aber in seiner Monographie indirekt ab: Appius habe an dem Antrittsessen als Salier teilgenommen. ${ }^{26}$ Da Lentulus Niger um das Jahr 70 v. Chr. ${ }^{27}$ Sempronia socrus eius zu seinem Antrittsschmaus einlud (Macr. 3.13.11), hätte Tiberius seinen socer einladen können, egal ob er ein Priestertum innehatte oder nicht. Eigentlich soll Appius aber am Anfang des Festmahles nicht einmal der künftige Schwiegervater des Tiberius gewesen sein, so daß man jenem das Augurat zuschreiben könnte, wenn man davon ausgehen könnte, daß es sich hierbei um einen Antrittsschmaus und auch um eine historische Nachricht handelt.
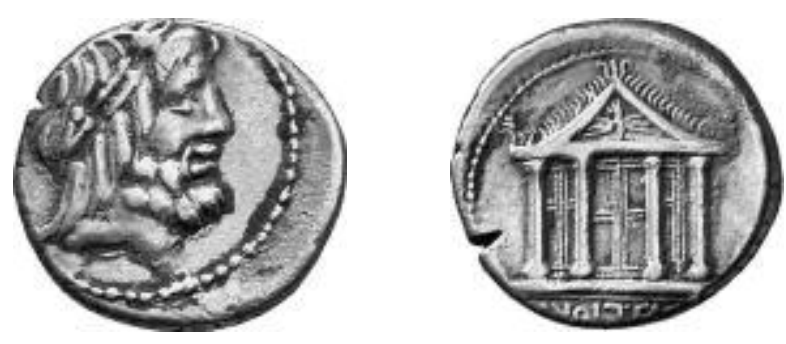

Der erste Denar des M. VOLTEI M. F.

Das Festessen wurde in der späteren Version an einem unbekannten Ort gegeben. Bebildern können wir aber die Originalfassung der Geschichte, denn ein gewisser M.

$<$ fiunt?>), gehandelt haben. Der Auftritt des betagten Kriegers wurde neulich besprochen (Evans 1999: 87-89, 92).

${ }^{23}$ Mommsen 1879: 2.493 A. 158; Münzer 1920: 268 A. 1.

${ }^{24}$ Bardt 1871; Münzer 1920: 268 A. 1.

${ }^{25}$ Drumann 1835: 2.181; Broughton 1986: 1.495, 2.547.

${ }^{26}$ Münzer 1920: 268 A. 1.

${ }^{27}$ Zum Datum s. Ryan 1994: 59-65. 
Volteius prägte wohl im Jahre 78 einen Denar, der auf der Vorderseite den bärtigen Kopf des Jupiter mit Lorbeerkranz, auf der Rückseite den Tempel desselben auf dem Kapitol mit verschlossenen Türen zeigt. ${ }^{28}$ Tatsächlich handelt es sich hierbei um „die erste ausführliche Gebäudedarstellung auf dem Silbergeld der Republik.“29 Es ist nicht reiner Zufall, daß das dargestellte Gebäude ,il più importante tempio della città di Roma“" ist. ${ }^{30}$ Serviert wurde das Essen in der Tempelzelle des Jupiter: Silbergefäße für drei dort stehende Tische sollen die Ogulnii am Anfang des dritten vorchristlichen Jahrhunderts als kurulische Ädilen präsentiert haben (L. 10.23.12: trium mensarum argentea vasa in cella Iovis). Das Kultbild aus gebranntem Ton schrieb Varro einem gewissen Vulca aus Veii zu (Plin. NH 35.157). ${ }^{31}$ Auch der Blitz in der Rechten des Standbildes war aus Ton (Ovid. Fast. 1.201-202: Iuppiter angusta vix totus stabat in aede / inque Iovis dextra fictile fulmen erat). Das Jupiterbild bzw. sein Gesicht wurde an Festtagen mit Zinnober bestrichen, also rot gefärbt (Plin. NH 33.111: Iovis ipsius simulacri faciem diebus festis minio inlini solitam, 35.157: fictilem eum fuisse et ideo miniari solitum; vgl. Cic. Fam. 9.16.8: miniati Iovis). L. Marcius weihte einen in Spanien erbeuteten, karthagischen Rundschild von Gold ${ }^{32}$ bzw. von Silber, ${ }^{33}$ der bis zum Brand am 6. Juli 83 über der Tür des Tempels aufgehängt war: isque clupeus supra fores Capitolinae aedis usque ad incendium primum fuit (Plin. NH 35.14). ${ }^{34}$ Die kurulischen Ädilen des Jahres 193, M. Aemilius Lepidus und L. Aemilius Paullus, finanzierten mit Bußgeld die vergoldeten Schilde, die sie am Giebel des kapitolinischen Tempels aufhängen ließen: ex ea pecunia clupea inaurata in fastigio Iovis aedis posuerunt (L. 35.10.12). Derselbe M. Aemilius Lepidus war Zensor in den Jahren 179-178. Aus dem ihnen zugewiesenen Geld führten die beiden Zensoren

\footnotetext{
${ }^{28}$ M. H. Crawford (1974: 1.399) entschied sich für das Prägejahr 78. Es ist W. Hollstein (1993: 19-22) gelungen, über den fünften Denar, eine Sonderprägung (SC), die sich auch durch die weitaus kleinere Stempelzahl von den vier übrigen Denaren der Serie abhebt, das von Crawford gebotene Prägedatum $78 \mathrm{zu}$ bestätigen.

${ }^{29}$ Fuchs 1969: 17.

${ }^{30}$ Tagliamonte 1996: 144.

${ }^{31}$ Siehe A. Rumpf (1961: 1226): „Die Frage, woher Varro den Namen des Künstlers V(ulca) erfahren hat, und woher er ihn überhaupt erfahren konnte, bleibt ungelöst.“

32 Da Gold und Vergoldung in den Quellen mitunter verwechselt werden, war das Weihgeschenk möglicherweise kein Goldschild, sondern ein vergoldeter; so Sehlmeyer 1999: 160 m. A. 110.

${ }^{33}$ F. Münzer (1897: 200-201) hielt den angeblich goldenen Schild (Plin. NH 35.14: ex auro) für einen silbernen. Den Beweis hierfür glaubte er in derselben Passage des Plinius gefunden zu haben, denn ebenda werden Schilde erwähnt, die zwar nicht als silbern betrachtet wurden, in Wirklichkeit aber es waren: docuerit...argenteos esse clupeos (NH 35.14). Der Plural hätte ihn davon abhalten sollen, denn Plinius kannte einen einzigen karthagischen Prachtschild: talem Hasdrubalis invenit Marcius $(\mathrm{NH}$ 35.14). Der Weg zu einem silbernen Schild ist kürzer. Livius beschreibt den Schild als silbern da, wo er dem Claudius Quadrigarius allein folgt: praedam ingentem partam; in ea fuisse clipeum argenteum pondo centum triginta septem cum imagine Barcini Hasdrubalis (L. 25.39.12-13). Er erwähnt das Material nicht mehr da, wo er mehreren Gewährsmännern folgt: monimentumque victoriae eius de Poenis usque ad incensum Capitolium fuisse in templo clipeum Marcium appellatum cum imagine Hasdrubalis (L. 25.39.17). Aller Wahrscheinlichkeit nach war der clipeus „Marcius“ weder golden noch vergoldet, sondern silbern.

${ }^{34}$ In seiner Behandlung der Maßnahmen der Zensoren von 179 gegen Beutestücke auf dem Kapitol machte Sehlmeyer (1999: 161 m. A. 114-115) auf zwei Reden des älteren Cato aufmerksam: In der einen, Uti praeda in publicum referatur, bezog er Stellung gegen die Anbringung von Spolien an Privathäusern, in der anderen, Ne spolia figerentur nisi de hoste capta, beanstandete er die unrechtmäßige Anbringung von Spolien. Warum denn hat weder Cato noch sein Nachfolger den Schild entfernt, den Marcius in Spanien lediglich gefunden (Plin. NH 35.14: invenit) hatte? Der etwas komische Wortlaut der Pliniusstelle scheint die Antwort preiszugeben: in castris certe captis talem (sc. clipeum) Hasdrubalis invenit Marcius, Scipionum in Hispania ultor (NH 35.14). Der gefundene Rundschild durfte als ein weggenommener gelten, weil das Lager eingenommen worden war.
} 
getrennt mehrere Arbeiten durch (L. 40.51.2: opera ex pecunia attributa divisaque inter se haec fecerunt). Lepidus allein kümmerte sich um den Jupitertempel: aedem Iovis in Capitolio columnasque circa poliendas albo locavit et ab his columnis, quae incommode opposita videbantur, signa amovit clipeaque de columnis et signa militaria adfixa omnis generis dempsit (L. 40.51.3). ${ }^{35}$ Zur Zeit des imaginären Festessens in den 180er Jahren wären die Spolien schon so angebracht gewesen, daß sie störten (incommode opposita). Im Giebelfeld befand sich ein tönernes Viergespann (Fest. 342L: fictilium quadrigarum, quae erant in fastigio Iovis templi; Plin. $\mathrm{NH}$ 28.16: in fastigium eiusdem delubri praeparatae quadrigae fictiles, $\mathrm{NH} 35.157$ : fictiles in fastigio templi eius quadrigas), das man Vulca (Plin. NH 35.157) bzw. einem ungenannten vejentischen Künstler (Fest. 342L) zuschrieb. Aus Ton war zunächst auch die Statue des Summanus, „ein besonderer Gott des nächtlichen Himmels und der nächtlichen Blitze ${ }^{\text {‘36. }}$ : cum Summanus in fastigio Iovis optumi maxumi, qui tum erat fictilis, e caelo ictus esset nec usquam eius simulacri caput inveniretur, haruspices in Tiberim id depulsum esse dixerunt, idque inventum est eo loco, qui est ab haruspicibus demonstratus (Cic. Div. 1.16). Dem tum in dem Relativsatz qui tum erat fictilis darf man entnehmen, daß auch zur Zeit der Abfassung der Schrift ein simulacrum des Summanus noch vorhanden, nicht mehr aber tönern war. Es steht damit fest, daß Q. Lutatius Catulus, curator restituendi Capitolii (Varro ap. Gell. 2.10.2), ${ }^{37}$ ein nichttönernes Standbild des Summanus oben anbringen ließ. Die Tonstatue könnte indes schon früher ersetzt worden sein, etwa nach der hier von Cicero erwähnten Begebenheit. Getroffen wurde das alte Tonbild von einem Blitze in

\footnotetext{
${ }^{35}$ Diese Stelle macht dem Leser Unannehmlichkeiten. Die Lexika gehen nicht sehr ausführlich hierauf ein. Vgl. Richardson (1992: 222): "In 179 B.C. the walls and columns were restuccoed"; Tagliamonte (1996: 146): "rivestendo con stucchi le pareti e le colonne dell'edificio." Nach der anfänglichen Erwähnung der Säulen (columnasque) werden die Säulen noch zweimal erwähnt und es werden signa zweimal erwähnt. Über sie schreibt Sehlmeyer (1999: 160): "Signa bedeutet hier wahrscheinlich nicht Statuen" - dies meinte aber noch Richardson (1992: 222) — "denn sie werden wie die clipea von den

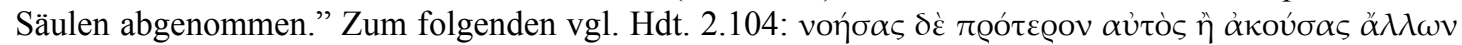
$\lambda \varepsilon \dot{\gamma} \omega$. Auf den ersten Blick scheinen zwei Arten von signa vorhanden gewesen zu sein, signa und signa militaria. Der Tempel war aber bekanntlich "il punto di arrivo delle processioni trionfali" (De Angeli 1996: 152), was nahe legt, daß hier keine nichtmilitärischen signa angebracht waren. Allenfalls gab es zwei Arten von militärischen signa, die an den Säulen befestigten signa und die offenbar an den Wänden befestigten signa militaria...omnis generis. Die Formulierung ab his columnis fällt auf: Abgenommen wurden die signa nicht von sämtlichen Säulen, sondern von “diesen.” Es handelt sich um die Säulen, die mit Kalk geglättet wurden, und das waren nur die äußeren Säulen ( circa). Die Vorhalle hatte drei Reihen von je sechs Säulen, die einfachen Säulenhallen neben der Cella der Iuno und der der Minerva hatten je drei Säulen (s. LTUR 3, Fig. 98). Insgesamt gab es also 24 Säulen, 16 äußere und 8 innere. In der Passage des Livius werden die 8 inneren Säulen übergangen: In der Formulierung clipeaque de columnis werden die Worte de columnis nicht deswegen hinzugefügt, weil diese Säulen andere waren als die schon erwähnten, sondern um die Aussage amovit clipea einzuschränken. Die Rundschilde wurden also nicht allesamt entfernt, sondern nur die an den Säulen befestigten: Schließlich bescheinigen uns die Quellen, daß der Marcius über der Tür blieb, und es ist davon auszugehen, daß der Zensor M. Aemilius die Schilde nicht beseitigte, die der kurulische Ädil M. Aemilius mit seinem Kollegen am Giebel aufhängen ließ. Streng genommen ist es möglich, daß signa und clipea auch an den 8 inneren Säulen befestigt waren, aber damals nicht entfernt wurden, weil die fraglichen Säulen nicht mit Weiß geglättet wurden. Der Grund, weswegen alles von den äußeren Säulen abgenommen wurde, incommode opposita, hätte aber auch bei den inneren Säulen gegolten. Aller Wahrscheinlichkeit nach war von vornherein nichts an den inneren Säulen befestigt gewesen. Nach $179 \mathrm{v}$. Chr. war nichts mehr an den äußeren Säulen befestigt. Von da an waren signa nirgends, clipea ihrerseits nur noch an dem Tempelgebäude selbst angebracht.

${ }^{36}$ Wissowa 1912: 135.

${ }^{37}$ Er scheint diese Stelle um die 16 bzw. 17 Jahre lang innegehabt zu haben; s. Ryan 1998: 304 A. 79.
} 
einem der drei Jahre 277-275 v. Chr. ${ }^{38}$ Das Inhaltsverzeichnis des Livius erzählt wie Cicero von der Bergung des caput durch die Haruspices. Sonst hätte man die zwei Stellen nicht in Zusammenhang miteinander gebracht, denn der Verfasser des Inhaltsverzeichnisses läßt die falsche Statue enthauptet werden: cum inter alia prodigia fulmine deiectum esset in Capitolio Iovis signum, caput eius per haruspices inventum est (L. Per. 14). Zunächst vermutet man, daß Livius dem Irrtum, der in der Zusammenfassung vorliegt, keinesfalls erlegen sein könnte. In dem zehnten Buch desselben finden wir jedoch einen Bericht über die ebenda auf das Jahr 296 gesetzte kurulische Ädilität der Ogulnii, die sich unter anderem auch um den Jupitertempel gekümmert haben sollen. Es heißt: aenea in Capitolio limina et trium mensarum argentea vasa in cella Iovis Iovemque in culmine cum quadrigis...posuerunt (L. 10.23.12). Die Notiz Iovemque in culmine cum quadrigis ist verdächtig. Es überrascht, daß Livius das Viergespann auf die Ogulnii zurückführt, denn es wird sonst, wie wir gesehen haben, in Verbindung mit den Vejentern gebracht. Zu dem Zeitpunkt, als Livius es anbringen läßt, war es nach den anderen Quellen schon gut zwei Jahrhunderte lang vorhanden. ${ }^{39}$ Stutzig macht uns auch die Angabe in culmine, denn nach den anderen Quellen befand sich das Viergespann in fastigio. Freilich läßt Cicero auch das Standbild des Summanus in fastigio sein. ${ }^{40}$ Wir verfügen offenbar über keine Quelle, die die beiden Götterstandbilder erwähnt und sie aufeinander bezieht. ${ }^{41}$ Trotzdem wird man annehmen dürfen, daß sich das tönerne Viergespann im Giebelfeld, das Tonbild des Summanus seinerseits auf dem First befand, denn es war ja das Summanusbild, das vom Blitz getroffen wurde. Die Richtigkeit der livianischen

\footnotetext{
${ }^{38}$ Gesetzt wurde dieses Ereignis auf d. J. 278 v. Chr. von Wissowa (1912: 135), während es von anderen auf d. J. 275 v. Chr. gesetzt worden ist: Weinstock 1931: 897-98; Richardson (1992: 222): ,a statue of Summanus, the head of which was broken off by lightning in 275 B.C."; Tagliamonte (1996: 146): „Nel 275 a.C. un fulmine colpì la statua acroteriale di Summanus.“ Das J. 278 ist zu früh, das J. 275 zu präzis. Es geht aus L. Per. 13 hervor, daß das Konsulatsjahr des C. Fabricius (278 v. Chr.) im 13. Buch des Livius behandelt wurde. In L. Per. 14 wird erst nach dem Blitzschlag ein Konsul erwähnt, Curius Dentatus (275 v. Chr.). Der Blitzschlag gehört daher in eines der drei Konsulatsjahre 277-275 v. Chr. Datiert haben wir damit nicht nur die Beschädigung der Tonstatue des Summanus. Wissowas (1912: 135) Ansicht, ,zur Sühne für dieses Prodigium erhielt der Gott einen Tempel beim Circus maximus," ist zweifelsohne richtig. Der Tempel des Summanus, der selbst i. J. 197 vom Blitz getroffen wurde (L. 32.29.1), hatte seinen Ursprung in der Zeit, als die Römer den König Pyrrhus noch fürchteten: reddita, quisquis is est, Summano templa feruntur, / tum, cum Romanis, Pyrrhe, timendus eras (Ov. Fast. 6.731-732). Unter Berücksichtigung von L. Per. 14 zog F. Bömer (1958: 2.385) den Schluß, daß „278 als Gründungsjahr“ anzusehen ist; um ein Jahr zu früh beginnt der Zeitraum bei A. Ziolkowski (1992: 154): „,between 278 and 275.“Vgl. G. Radke (1979: 431): „278 v. Chr....geweiht“; Richardson (1992: 373): „built during the war with Pyrrhus.“ Hervorzuheben ist der Vorschlag des Ziolkowski (1992: 154): „If alia prodigia included the pestilence which ravaged Rome in 276, the temple would have been vowed that year." Es steht nicht fest, daß die aedes Summani schon bis zum J. 275 fertiggestellt und geweiht wurde, sie wurde aber mit Sicherheit in den J. 277-275 gelobt.

${ }^{39}$ Man sucht die Nachricht dadurch zu retten, daß man die Ogulnii das ursprüngliche Viergespann ersetzen läßt, und zwar durch eines aus kostbarem Metall. Vgl. Richardson (1992: 222): „This was replaced by another group in 296 B.C., the new one probably of bronze, because brazen thresholds and other luxuries were installed...at this time“; Tagliamonte (1996: 146): „Nel 296 a.C....la quadriga fittile di Iuppiter fu sostituita con una di bronzo." Nach aenea...limina und argentea vasa müßte man entgegen dieser Vermutung annehmen, daß kein wertvolles Metall für den Iovem...cum quadrigis verwendet wurde.

${ }^{40}$ Wenn man die Angabe in culmine als eine Präzisierung von in fastigio betrachtet, dann kann die Angabe in fastigio kaum falsch sein, denn das, was in culmine ist, ist auch in fastigio. Wenn also das simulacrum des Summanus auf dem First stand, wäre Ciceros Angabe Summanus in fastigio nicht als falsch, sondern als vage zu bewerten.

${ }^{41}$ C. Hülsen (1899: 1533) setzte weder das Viergespann noch das Summanusbild auf den First, sondern ließ beide für den Giebel bestimmt sein.
} 
Angabe in culmine scheinen auf den ersten Blick drei Zeilen des Plautus zu bestätigen: nam nunc ego si te surrupuisse suspicer / Iovi coronam de capite ex Capitolio, / qui in columine astat summo: si id non feceris (Plaut. Trin. 83-85). Plautus gibt indes nicht an, daß das Viergespann in columine aufgestellt war. ${ }^{42} \mathrm{Da}$ „der Trinummus eher nach als vor $190 \mathrm{v}$. Chr. zum erstenmal gegeben worden ist und somit unter die letzten Stücke des Dichters gehört, ${ }^{\text {"43 }}$ war der Tempel des Summanus zur Zeit der Aufführung des Trinummus schon um die 80 Jahre alt. Es kann sein, daß die Existenz eines Tempels des Summanus den Eindruck entstehen ließ, daß die Tonstatue auf dem First des kapitolinischen Tempels ein Jupiterbild sei. Es kann auch sein, daß Plautus selbst es besser wußte: Durch die Worte si id non feceris „wird der Diebstahl als fast unmöglich...hingestellt"; zu tun haben wir es mit einem „Beispiel einer aus der Luft gegriffenen Beschuldigung,“ die „,vielleicht sprichwörtlich“ ist. ${ }^{44}$ Die Angabe in der Zusammenfassung des Livius bleibt falsch, aber ist nachvollziehbar: Es begegnet hier, wie bei Plautus früher, die landläufige Meinung über die Statue auf dem First des kapitolinischen Tempels, während bei Cicero die Ansicht eines belesenen Mannes begegnet. ${ }^{45}$ Wenn die Ogulnii eine Statue in culmine aufstellten, dann die des Summanus. Sagen wir es einmal so: Auf den Blitz des besten und höchsten Jupiter ist weitaus mehr Verlaß als auf die hier in Rede stehenden literarischen Quellen. Das Giebelfeld sah auf jeden Fall anders aus als auf dem Denar des M. Volteius: „Das Blitzbündel im Pediment diente mit Sicherheit nur der

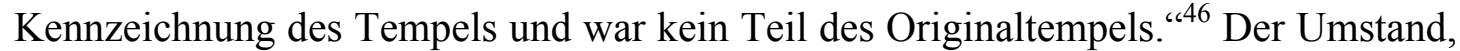
daß der kapitolinische Tempel hier viersäulig abgebildet ist, besagt also nicht, daß er einst viersäulig war, ${ }^{47}$ denn es ,könnten deshalb nur vier Säulen graviert worden sein, um durch sie die drei cellae für Jupiter, Juno und Minerva zu verdeutlichen." ${ }^{\text {48 }} \mathrm{Zum}$ Zeitpunkt der Prägung des Denars war nun der Tempel noch nicht wieder errichtet worden. Der Münzmeister konnte also den Tempel der ausgehenden Republik nicht abbilden, ${ }^{49}$ sondern wird die Wiederaufrichtung des Tempels gefeiert haben, ${ }^{50}$ indem er einen an den abgebrannten erinnernden Tempel darstellte. ${ }^{51}$

Streng genommen ist Appius nur als Mitglied der Priesterschaft der Salier bezeugt: sed quid loquor de histrionibus, cum Appius Claudius, vir triumphalis qui Salius ad usque senectutem fuit, pro gloria obtinuerit quod inter collegas optime saltitabat? (Macr. 3.14.14). Man wende nicht gegen die herkömmliche Identifizierung mit Appius ein, daß der Senat den von ihm beantragten Triumph nicht gewährt hat, denn

\footnotetext{
${ }^{42}$ Vielleicht vermutete Hülsen (1899: 1533) deswegen in dem Iovi des Plautus die Statue des Summanus.

${ }^{43}$ Conrad 1931: 44-45.

${ }^{44}$ Conrad 1931 z. St.

${ }^{45}$ Auf zwei Inschriften (ILS 3057-3058) aus Oberitalien kommt der Name Summanus als ein Epitheton Jupiters vor. Es wäre konziliatorisch, auch in der Tonstatue auf dem First Jupiter Summanus zu sehen, damit man sowohl Cic. Div. 1.16 als auch L. Per. 14 akzeptieren kann. Nachdem Summanus einen eigenen Tempel in Rom erhalten hatte, also zu Lebzeiten des Plautus, war Summanus in der Stadt Rom wohl kein Epitheton Jupiters mehr. Eine ara soll schon Titus Tatius Summano gelobt haben (Varro LL 5.74).

${ }^{46}$ Hollstein 1993: 11-12.

${ }^{47}$ P. V. Hill (1980: 41) schloß nicht aus, daß ,the first (Etruscan) temple...actually tetrastyle“ war.

${ }^{48}$ Hollstein 1993: 13; so auch Hill 1980: 41.

${ }^{49} \mathrm{Vgl}$. Crawford (1974: 1.400): ,Since the temple had been destroyed and not yet rebuilt at the time of this issue, the representation is not necessarily accurate."

${ }^{50}$ So Hollstein (1993: 13): Der Denar hat ,einen aktuellen, auf Sullas Restaurationstätigkeit ... bezogenen Hintergrund.“

${ }^{51}$ Vgl. Hill (1980: 41): „It is possible that the first temple is that shown on denarii of M. Volteius.“
} 
er feierte einen Triumph trotzdem, und der Ausdruck vir triumphalis bei Macrobius scheint dadurch gerechtfertigt zu sein. Da der vir triumphalis der geschickteste Tänzer im Kollegium war und dies ad usque senectutem, kann er kaum mit Russus (Cos. 268) identisch sein: Sein Triumph gilt als sicher, ${ }^{52}$ er starb aber während seines Amtsjahres und wird somit kein sehr hohes Alter erreicht haben. Es spricht nicht unbedingt gegen Caudex (Cos. 264), daß sein Triumph nicht in den Triumphalfasten angegeben ist, ${ }^{53}$ denn Eutrop (2.18.2: in Sicilia contra eos [sc. Afros] pugnatum est et Ap. Claudius de Afris et rege Siciliae Hierone triumphavit) zweifelte nicht an seinem Triumph, und Macrobius könnte ebenso die Notiz für richtig gehalten haben. Alle Wahrscheinlichkeit spricht aber dafür, daß eher der Konsul des Jahres 143 als der des Jahres 264 in die Geschichtsbücher eingetanzt hat, und da sein Konsulat in die Zeit nach der Verabschiedung des villischen Gesetzes gehört und er frühestens im elften Jahr nach seinem Konsulatsjahr starb, darf man ihm seine senectutem nicht absprechen. Ausgerechnet im Falle eines Salius braucht man die Möglichkeit, daß Appius diese Stellung mit dem Augurat kombinierte, nicht in Betracht zu ziehen: „Nur die Stellung des Saliers scheint mit der Bekleidung eines andern Priestertums unverträglich gewesen zu sein." ${ }^{54}$ Dieser Schluß basiert auf dem Mitgliederverzeichnis der Salii Palatini, von welchem Fragmente aus den Jahren 170-219 n. Chr. erhalten sind. Klose war bereit, diese Regel aufzuheben: „Appius Claudius war nach Plut. Ti. Gr. 4 auch Augur, folglich kann es nicht verboten gewesen sein, wie Wissowa...behauptet, gleichzeitig das Amt eines Saliers und eines der großen Priesterämter zu bekleiden. ${ }^{\text {"55 }}$ Auch wenn wir wüßten, daß Appius das Augurat erhielt, würde diese Tatsache die herrschende Ansicht noch lange nicht widerlegen: Es wäre ohne weiteres denkbar, daß er aus der Priesterschaft der Salier etwa 135 oder 134 ausschied, um Augur werden zu dürfen. Für die herrschende Ansicht spricht lediglich die Annahme, daß die Regel schon zur Zeit der Republik gültig war. Dagegen spricht das vermeintliche Augurat des Appius überhaupt nicht, da ein wirkliches mit der Regel vereinbar wäre. Das nirgends bezeugte Augurat, das schon Klose dazu verführte, die Regel aufzuheben, verführte Broughton später dazu, die tatsächlich bezeugte Salierwürde preiszugeben: „Since he could not remain a Salius after attaining a major priesthood, Claudius, even if he was once a Salius before becoming an augur..., cannot be the Ap. Claudius vir triumphalis in Macrob(ius)..., who gained fame by dancing as a Salius in his old age." ${ }^{56}$ Man darf die Identifikation mit dem vir triumphalis nicht zurückweisen und den Salierdienst trotzdem für möglich halten, denn es gibt keinen zweiten Beleg dafür, daß Appius die Würde des Saliers bekleidet hat. War er Salier, so wurde er einer vor dem Tode seines Vaters im Jahre 167, da nur der Sohn von noch lebenden Eltern in die Priesterschaft eintreten durfte. $^{57}$

Appius ist also nunmehr weder als Princeps Senatus noch als Augur anzusehen, er darf aber weiterhin als ein an Geschmeidigkeit nicht zu übertreffender Salier gelten. Die Plutarchstelle (Ti. Gr. 4.2) ist nunmehr als ein fünfter Beleg für den Vorrang des Africanus einzustufen, welcher versehentlich zusammen mit der Übereinkunft auf Appius übertragen worden ist. Appius gesellt sich eher zu den ,four men incorrectly

\footnotetext{
${ }^{52}$ Broughton 1986: 1.200 .

${ }^{53}$ Vgl. Broughton 1986: 1.203.

${ }^{54}$ Wissowa 1912: 486 A. 6, 493-94.

${ }^{55}$ Klose 1910: 52.

${ }^{56}$ Broughton 1986: 3.56 .

${ }^{57}$ Klose 1910: 50, 52; Broughton 1986: 1.436.
} 
and intentionally attested“58 als zu den „two men incorrectly but inadvertently attested" als Princeps Senatus. ${ }^{59}$ Eine Passage einer Rede Ciceros, in der sich dieser an Clodius wendet, ist hier von Belang. Als er von der Ausstoßung aus dem Senate sprach, die Ap. Claudius (Cos. 79), der Vater des Clodius, hinnehmen mußte, beschrieb er ihn, den Sohn des Konsuls des Jahres 143, als den Sohn eines hochangesehenen Mannes (Cic. Dom. 84: patrem tuum, civem optimum, clarissimi viri filium...L. Philippus censor avunculum suum praeteriit in recitando senatu). Hätte Cicero überhaupt vom Prinzipat des Appius gewußt, so hätte er das Wissen davon an dieser Stelle verraten müssen. Vor kurzem hätte man Ciceros Unkenntnis über das Prinzipat als unerwartet einstufen müssen und unter den Teppich kehren wollen. Gelassen dürfen wir aber jetzt in Ciceros Unwissenheit eine Bestätigung sehen, daß es hierüber gar nichts zu wissen gegeben hat. ${ }^{60}$

Dr. Francis X. Ryan

Technische Universität Dresden, Institut für Geschichte

E-mail:fxryan@gmx.de

\footnotetext{
${ }^{58}$ Rullianus macht sechs; s. Ryan 2003: 112.

59 Vgl. Ryan 1998: 222. Bei den beiden, versehentlich bezeugten Principes Senatus (s. Ryan 1998: 203-209) handelt es sich um einen Irrtum der Abschreiber der Texte.

${ }^{60}$ Der Verfasser bedankt sich bei Herrn Prof. Dr. M. Jehne und Herrn Prof. Dr. J. von UngernSternberg, die eine Vorgängerversion des Aufsatzes freundlicherweise durchsahen; für die Vergabe eines Forschungsstipendiums hat er der Alexander von Humboldt-Stiftung zu danken.
}

Abbildungsnachweis 


\section{Bibliographie}

Bardt, C. (1871) Die Priester der vier grossen Collegien aus römisch-republikanischer Zeit. Berlin: Trowitzsch.

Bernstein, F. (1998) Ludi publici. Untersuchungen zur Entstehung und Entwicklung der öffentlichen Spiele im republikanischen Rom. Stuttgart: Steiner (Historia Einzelschriften; 119.)

Bömer, F. (1958) P. Ovidius Naso, Die Fasten. Heidelberg: Winter.

Broughton, T. R. S. (1986) The Magistrates of the Roman Republic. Atlanta: Scholars Press (Reprint d. Ausg. New York, 1951-1952.)

Burckhardt, L. - Ungern-Sternberg, J. von (1994) ,Cornelia, Mutter der Gracchen.' - Reine Männersache? Frauen in Männerdomänen der antiken Welt, hg. v. M. H. Dettenhofer. Köln: Böhlau, 97-132.

Conrad, F. (1931) Ausgewählte Komödien des T. Maccius Plautus. Trinummus. 6. Aufl. Leipzig: Teubner.

Crawford, M. H. (1974) Roman Republican Coinage. Cambridge: Cambridge University Press 1974.

De Angeli, S. (1996) ,Iuppiter Optimus Maximus Capitolinus, Aedes (fasi tardorepubblicane e di età imperiale).' — LTUR 3, 148-53.

Drumann, W. K. A. (1835) Geschichte Roms in seinem Uebergange von der republikanischen zur monarchischen Verfassung, oder Pompejus, Caesar, Cicero und ihre Zeitgenossen, nach Geschlechtern und mit genealogischen Tabellen. Koenigsberg: Bornträger.

Evans, R. J. (1999) ,Displaying Honourable Scars: A Roman Gimmick.' - $\mathrm{ActaCl}$ 42, 77-94.

Fuchs, G. (1969) Architekturdarstellungen auf römischen Münzen der Republik und der frühen Kaiserzeit. Berlin: de Gruyter.

Hill, P. V. (1980) ,Buildings and Monuments of Ancient Rome on Republican Coins, c. 135-40 BC.' - RIN 82, 33-52.

Hollstein, W. (1993) Die stadtrömische Münzprägung der Jahre 78-50 v. Chr. zwischen politischer Aktualität und Familienthematik. München: tuduv.

Hülsen, C. (1899) ,Capitolium.' - RE 3, 1531-38.

Klose, A. (1910) Römische Priesterfasten. Diss. Breslau.

Konrad, C. F. (1989) ,Livy on the Betrothal of Cornelia Gracchi [38.57.7].' — Philologus $133,155-57$.

Mommsen, T. $(1864,1879)$ Römische Forschungen. Berlin: Weidmann.

Mommsen, T. (1887-88) Römisches Staatsrecht. Leipzig: Hirzel.

Münzer, F. (1897) Beiträge zur Quellenkritik der Naturgeschichte des Plinius. Berlin: Weidmann.

Münzer, F. (1899), Claudius 295.' - RE 3, 2848.

Münzer, F. (1920) Römische Adelsparteien und Adelsfamilien. Stuttgart: Metzler.

Radke, G. (1979) ,Summanus.' - Kl. P. 5, 431.

Richardson, L., Jr. (1992) A New Topographical Dictionary of Ancient Rome. Baltimore: Johns Hopkins University Press.

Rumpf, A. (1961), Vulca.' - RE 9A, 1223-26.

Ryan, F. X. (1994) ,The Aedileship of P. Sulpicius Galba.' - Eos 82, 55-65.

Ryan, F. X. (1998) Rank and Participation in the Republican Senate. Stuttgart: Steiner. 
Ryan, F. X. (2002), Varro bei Varro? Der Brief an Oppianus und das Handbuch für Pompeius.' - Hommages à Carl Deroux II: Prose et linguistique, médecine, éd. par Pol Defosse. Bruxelles: Latomus, 372-85.

Ryan, F. X. (2003) ,Die Fasten der Erstgewählten des Senates.' - Hyperboreus 9, $112-13$.

Sehlmeyer, M. (1999) Stadtrömische Ehrenstatuen der republikanischen Zeit. Stuttgart: Steiner (Historia Einzelschriften; 130.)

Sumner, G. V. (1973) The Orators in Cicero's Brutus: Prosopography and Chronology. Toronto: University Press.

Tagliamonte, G. (1996) ,Iuppiter Optimus Maximus Capitolinus, Aedes, Templum (fino all'a. 83 a.C.).' - LTUR 3, 144-48.

Valgiglio, E. (1963) Plutarco, Vita dei Gracchi. Roma: Signorelli.

Weinstock, St. (1931) ,Summanus.' - RE 4A, 897-98.

Willems, P. (1878) Le Sénat de la république romaine. Louvain: Peeters.

Wissowa, G. (1912) Religion und Kultus der Römer. 2. Aufl. München: Beck.

Ziolkowski, A. (1992) The Temples of Mid-Republican Rome and their Historical and Topographical Context. Roma: "L'Erma" di Bretschneider. 